\title{
Terahertz phase slips in striped $\mathrm{La}_{2-x} \mathrm{Ba}_{x} \mathrm{CuO}_{4}$
}

\author{
D. Fu $\odot,{ }^{1}$ D. Nicoletti $\odot,{ }^{1}$ M. Fechner, ${ }^{1}$ M. Buzzi $\odot,{ }^{1}$ G. D. Gu, ${ }^{2}$ and A. Cavalleri ${ }^{1,3, *}$ \\ ${ }^{1}$ Max Planck Institute for the Structure and Dynamics of Matter, 22761 Hamburg, Germany \\ ${ }^{2}$ Condensed Matter Physics and Materials Science Department, Brookhaven National Laboratory, Upton, New York 11973, USA \\ ${ }^{3}$ Department of Physics, Clarendon Laboratory, University of Oxford, Oxford OX1 3PU, United Kingdom
}

(Received 16 September 2021; revised 2 December 2021; accepted 3 January 2022; published 18 January 2022)

Interlayer transport in high- $T_{C}$ cuprates is mediated by superconducting tunneling across the $\mathrm{CuO}_{2}$ planes. For this reason, the terahertz frequency optical response is dominated by one or more Josephson plasma resonances and becomes highly nonlinear at fields for which the tunneling supercurrents approach their critical value $I_{C}$. These large terahertz nonlinearities are in fact a hallmark of superconducting transport. Surprisingly, however, they have been documented in $\mathrm{La}_{2-x} \mathrm{Ba}_{x} \mathrm{CuO}_{4}$ (LBCO) also above $T_{C}$ for doping values near $x=\frac{1}{8}$ and interpreted as an indication of superfluidity in the stripe phase. Here, electric-field-induced second harmonic is used to study the dynamics of time-dependent interlayer voltages when LBCO is driven with large-amplitude terahertz pulses, in search of other characteristic signatures of Josephson tunneling in the normal state. We show that this method is sensitive to the voltage anomalies associated with $2 \pi$ Josephson phase slips, which near $x=\frac{1}{8}$ are observed both below and above $T_{C}$. These results document a regime of nonlinear transport that shares features of fluctuating stripes and superconducting phase dynamics.

DOI: 10.1103/PhysRevB.105.L020502

The $c$-axis terahertz-frequency electrodynamics of high$T_{C}$ cuprates [1] can be described by stacks of extended Josephson junctions [2,3] with distributed tunneling inductance $L_{J}(x, y, t)$ between capacitively coupled planes $(x$ and $y$ are, here, the in-plane spatial coordinates, and $t$ is the time). For low fields, $L_{J}=\frac{\hbar}{2 e I_{C}}$ (where $e$ is the electron charge and $I_{C}$ the critical current of the junction) is independent of space and time, and a Josephson plasma resonance (JPR) is found at $\omega_{\mathrm{JPR}}=2 \pi / \sqrt{L_{J} C}$ ( $C$ is the equivalent capacitance of the planes). In most cuprates, $\omega_{\mathrm{JPR}}$ ranges between gigahertz [4,5] and terahertz [6] frequencies. As characteristic for a plasmonic response, for $\omega>\omega_{\mathrm{JPR}}$, the superconductor is transparent, corresponding to a positive dielectric function $\varepsilon_{1}(\omega)>0$, whereas for $\omega<\omega_{\mathrm{JPR}}$, it is a perfect reflector with $R=1$ and $\varepsilon_{1}(\omega)<0$.

At high electric fields, the Josephson electrodynamics become nonlinear [7-9]. As radiation at photon energies below the average superconducting gap couples weakly to the order parameter amplitude $|\psi(x, y, t)|$, the electrodynamics are primarily determined by changes of the order parameter phase. The phase difference between adjacent layers $\Delta \varphi$ (henceforth referred to simply as $\varphi$ ) is therefore the relevant parameter, and the voltage that develops across the planes is expressed,

\footnotetext{
*andrea.cavalleri@mpsd.mpg.de
}

Published by the American Physical Society under the terms of the Creative Commons Attribution 4.0 International license. Further distribution of this work must maintain attribution to the author(s) and the published article's title, journal citation, and DOI. Open access publication funded by the Max Planck Society. according to the second Josephson equation, as $V=\frac{\hbar}{2 e} \frac{\partial \varphi}{\partial t}$ [see Fig. 1(a)].

For an intuitive understanding of these physics, we consider here the case of a Josephson junction under a DC current bias, $I$ [10]. In this condition, the phase dynamics of the junction can be modeled as the motion of a fictitious particle in the "washboard" potential $U(\varphi)=-\frac{\hbar I_{C}}{2 e}\left(\cos \varphi+\frac{I}{I_{C}} \varphi\right)$, sketched in Fig. 1(b) [11]. These dynamics are well understood, both in the classical and in the quantum regime $[12,13]$. For $I \ll I_{C}$, the potential $U$ has local minima where the phase particle is trapped and oscillates at the JPR frequency (blue). An increase of $I$ has the effect of tilting the potential and decreasing the barrier between two neighboring minima, progressively entering a nonlinear regime (red). For $I \sim I_{C}$, the phase "escapes" from the well, and a net voltage develops at the edges of the junction (green). By decreasing the bias current, the potential tilt is reduced, and the particle will be retrapped in the new potential minimum [10].

A more comprehensive description of the effect of an intense transient terahertz field on the extended Josephson coupled cuprate planes (i.e., with dimensions that exceed the London penetration depth) can be obtained by simulating the electrodynamics with the sine-Gordon equation $[14,15]$, which in one dimension reads

$$
\frac{\partial^{2} \varphi(x, t)}{\partial t^{2}}+2 \gamma \frac{\partial \varphi(x, t)}{\partial t}+\omega_{\mathrm{JPR}}^{2} \sin \varphi(x, t)=\frac{c^{2}}{\varepsilon} \frac{\partial^{2} \varphi(x, t)}{\partial x^{2}} .
$$

Here, $x$ is the in-plane coordinate along the propagation direction of the pulse, $\varepsilon$ the relative dielectric permittivity, $c$ the speed of light in vacuum, and $\gamma$ a damping coefficient which accounts for the tunneling of normal quasiparticles.

Representative results of these simulations are shown in Fig. 1(c), where we report the time- and space-dependent 
(a)

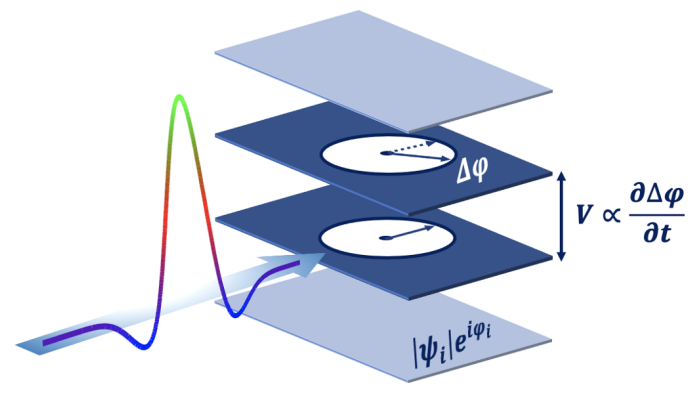

(b)

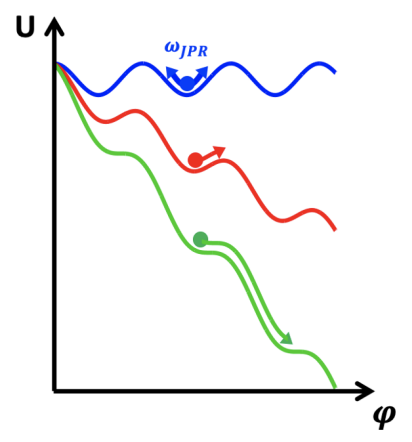

(c)
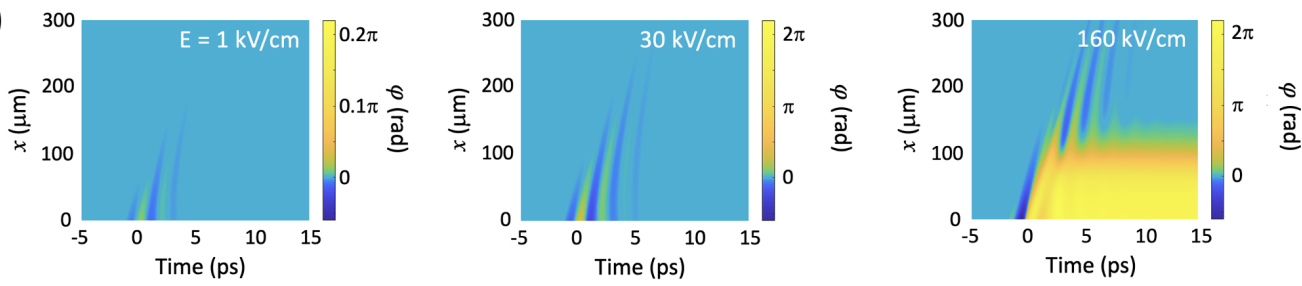

(d)
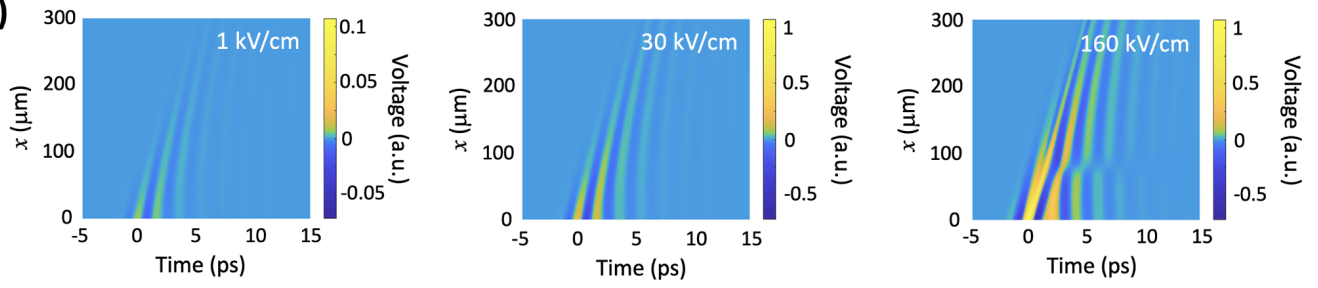

(e)

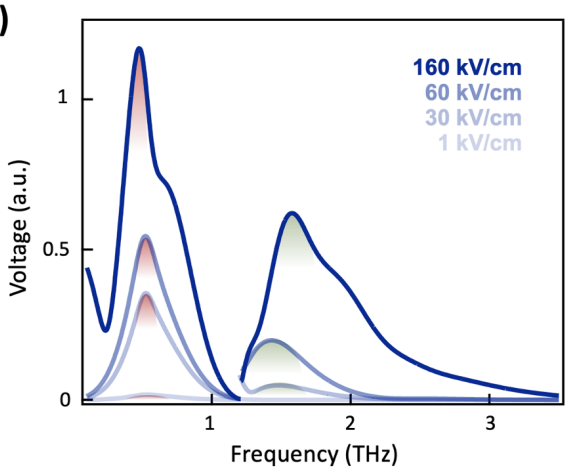

(f)

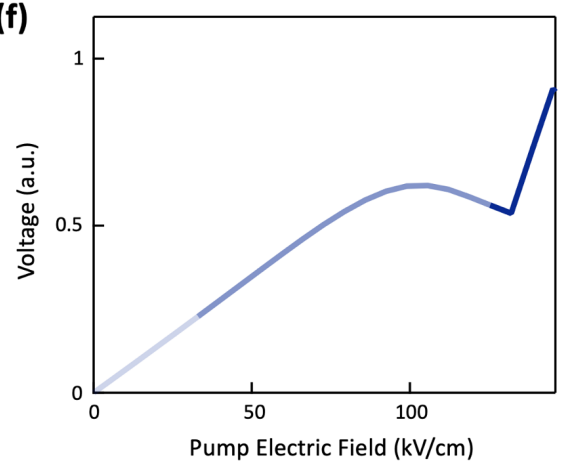

FIG. 1. (a) Schematic representation of a layered superconductor in the presence of a single-cycle terahertz driving field polarized along the out-of-plane direction. Depending on the instantaneous electric field carried by the pulse (color coded from blue through red to green), deformations of different magnitude in the space- and time-dependent order-parameter phase $\varphi$ are achieved. According to the second Josephson equation, these phase gradients lead to the development of a voltage $V$ across the layers. (b) Such a scenario can be visualized in terms of a Josephson junction under the effect of a current bias, characterized by a tilted washboard potential. Each value of the electric field carried by the pulse corresponds to a certain tilting amplitude. Depending on the peak electric field, different regimes can be explored, starting from the linear one (blue), characterized by a response at the Josephson plasma frequency $\omega_{\mathrm{JPR}}$ to a nonlinear perturbative regime (red), until approaching the critical current (green), a condition in which one expects to observe phase slips. (c) Calculated space- and time-dependent interlayer phase $\varphi(x, t)$ for a single extended Josephson junction illuminated by a terahertz pulse as that in (a), with a spectrum in resonance with $\omega_{\text {JPR }}$ and different terahertz peak field values $E_{\text {peak }}=1,30$, and $160 \mathrm{kV} / \mathrm{cm}$. Here, $x$ denotes the in-plane direction along the pulse propagation. In the rightmost panel, the occurrence of a phase slip is apparent. (d) Corresponding space- and time-dependent interlayer voltages $V(x, t) \propto \partial \varphi(x, t) / \partial t$. (e) Fourier transforms of the voltages in (d), calculated at the junction boundary $(x=0)$ for different $E_{\text {peak }}$ values. The peaks at $\sim \omega_{\text {drive }}$ and $\sim 3 \omega_{\text {drive }}$ are shaded in red and green, respectively. All spectra at $\omega>1.2 \mathrm{THz}$ have been multiplied by 30 . (f) Terahertz electric-field-dependent voltage, estimated at the peak frequency in (d) by scanning $E_{\text {peak }}$ continuously. A marked jump indicative of a phase slip is apparent. 
Josephson phase in the presence of an out-of-plane polarized driving field, with the same pulse shape shown in Fig. 1(a) and a spectrum peaked at $\omega_{\mathrm{JPR}}=0.5 \mathrm{THz}$. The plots from left to right refer to increasing values of the peak field. For small electric fields and thus small phase, $\sin \varphi(x, t) \simeq \varphi(x, t)$, and the sine-Gordon equation yields a linear wave equation, leading to Josephson plasma waves (left panel). For progressively increasing fields, larger phase excursions are achieved, and the nonlinear regime is accessed. Here, the oscillation frequency exhibits a redshift caused by the higher order terms in the $\sin \varphi$ expansion. Concomitantly, a rich phenomenology occurs, including odd harmonic generation and parametric amplification of Josephson plasma waves, which have been experimentally verified in a number of cuprate superconductors [16-19].

As the current of the junction reaches the critical value $I_{C}, 2 \pi$ phase slips are expected to develop across the junction, as already qualitatively captured by the analogy with the washboard potential depicted in Fig. 1(b). In the right panel of Fig. 1(c), the occurrence of a phase slip in the material is apparent.

It should be noted here that, for the damping values $\gamma$ and the shape of the terahertz pulse used for our experiments and included in these simulations, we do not expect soliton modes like those reported in Ref. [17]. Therein, these could be excited because the phase was driven with different terahertz pulse shapes, that is, narrowband multicycle pulses from a free-electron laser.

As shown in Figs. 1(d)-1(f), evidence for phase slips is to be found if one can measure the voltage of the junction $V=\frac{\hbar}{2 e} \frac{\partial \varphi}{\partial t}$. Figure 1(e) shows the Fourier transform of the calculated Josephson voltage time profiles of Fig. 1(d), determined at the edge of the junction, i.e., for $x=0$. In the sample, this point corresponds to the vacuum-material interface. As expected, the linear response exhibits a spectrum peaked at the driving frequency $\omega_{\text {drive }} \simeq \omega_{\mathrm{JPR}}$. In the nonlinear regime $(30-60 \mathrm{kV} / \mathrm{cm})$ odd harmonics develop at $3 \omega_{\text {drive }}$ and $5 \omega_{\text {drive }}$, and these high-frequency components grow dramatically when approaching the phase-slip regime $(160 \mathrm{kV} / \mathrm{cm})$. A clear signature of the phase slip is evident in the peak voltage, determined at the fundamental frequency $\omega_{\text {drive }}$ as a function of the driving electric field [Fig. 1(f)]. Here, one observes an abrupt, discontinuous increase of the signal, which we shall take as a univocal fingerprint of the occurrence of a Josephson phase slip in the material.

We set out to measure the field scaling of the $c$-axis Josephson voltage, which develops at the surface of high- $T_{C}$ superconductors of the $\mathrm{La}_{2-x} \mathrm{Ba}_{x} \mathrm{CuO}_{4}$ (LBCO) family, when driven by strong field single-cycle terahertz pulses. $\mathrm{LBCO}$ is an extensively studied 214 cuprate, exhibiting an anomalous suppression of the transition temperature $T_{C}$ for doping levels $\sim \frac{1}{8}$ [see schematic phase diagram in the inset of Fig. 2(c)] [20], related to the formation of "stripes", a peculiar chargeand spin-order pattern within the $\mathrm{CuO}_{2}$ planes, consisting of one-dimensional chains of doped holes separated by antiferromagnetically ordered regions [21]. Recent studies [22] support the existence of a striped superfluid state at $T>T_{C}$ with a spatially modulated superconducting order parameter, a so-called pair-density-wave (PDW) state [23-25]. However, superfluid stripes are difficult to detect with conventional tech- niques (e.g., scanning tunnelling microscopy), which are not sensitive to the order parameter phase, nor are they visible in linear $c$-axis optical measurements, due to their crossalignment in neighboring $\mathrm{CuO}_{2}$ layers [see, e.g., the inset of Fig. 3(c)] [24].

A recent experiment on $\mathrm{LBCO}$ showed that this frustration is removed in the nonlinear optical response [19]. A giant terahertz third harmonic, characteristic of nonlinear Josephson tunneling, was observed in $\mathrm{La}_{1.885} \mathrm{Ba}_{0.115} \mathrm{CuO}_{4}$ above the superconducting transition temperature and up to the chargeordering temperature $T_{\mathrm{CO}}$. Such a response was modeled by assuming the presence of a PDW condensate, in which nonlinear mixing of optically silent tunneling modes drives large dipole-carrying supercurrents.

Here, we make use of an experimental probe technique to confirm the generation of odd harmonics of the phase and to measure voltage signatures of Josephson phase dynamics.

As in Ref. [19], we studied two compounds: $\mathrm{La}_{1.905} \mathrm{Ba}_{0.095} \mathrm{CuO}_{4}$ (LBCO 9.5\%), where the stripes are weaker and are only present below $T_{\mathrm{CO}} \simeq T_{\mathrm{SO}} \simeq T_{C} \simeq 32 \mathrm{~K}$ (here, $T_{\mathrm{CO}}$ and $T_{\mathrm{SO}}$ are the charge- and spin-ordering temperature, respectively), and $\mathrm{La}_{1.885} \mathrm{Ba}_{0.115} \mathrm{CuO}_{4}$ (LBCO $11.5 \%$ ), for which the superconducting transition is highly depleted $\left(T_{C} \simeq 13 \mathrm{~K}\right)$ and the stripe phase is far more robust $\left(T_{\mathrm{CO}} \simeq 53 \mathrm{~K}\right.$ and $\left.T_{\mathrm{SO}} \simeq 40 \mathrm{~K}\right)[20]$.

The single-cycle terahertz pump pulses were generated in $\mathrm{LiNbO}_{3}$ by the tilted-pulse front technique [26] with a spectrum peaked at $\sim 0.5 \mathrm{THz}$ and were focused at normal incidence on the sample surface, with polarization along the out-of-plane crystallographic axis and maximum peak fields up to $\sim 165 \mathrm{kV} / \mathrm{cm}[15]$.

The electric-field-induced second harmonic (EFISH) [27] was sampled by 100 -fs-long near-infrared (NIR) probe pulses with $800 \mathrm{~nm}$ wavelength, which were also polarized along the $c$ axis, and scanned in time through the profile of the terahertzfrequency pump. In equilibrium and without the pump, no second harmonic was found.

The pump-induced $400 \mathrm{~nm}$ second harmonic intensity $I_{\mathrm{SHG}}$ generated at the surface was detected with a photomultiplier. By scanning the time delay between the terahertz pump and NIR probe, the full second harmonic temporal profile could be determined.

The EFISH originates from a four-wave process involving the driving field $E_{\mathrm{THz}}$ and the NIR probe field $E_{\text {NIR }}$. A third-order nonlinear polarization $P^{(3)}(\omega)=$ $\chi^{(3)}\left(\omega_{\mathrm{NIR}}, \omega_{\mathrm{NIR}}, \omega_{\mathrm{THz}}\right) E_{\mathrm{NIR}} E_{\mathrm{NIR}} E_{\mathrm{THz}}$ is generated at $2 \omega_{\mathrm{NIR}} \pm$ $\omega_{\mathrm{THz}}$ and measured by the detector as $I_{\mathrm{SHG}} \propto\left|P^{(3)}(\omega)\right|^{2}$. In our setup, a passive second harmonic generated in the setup mixes with $P^{(3)}(\omega)$, allowing for heterodyne detection of a signal which is directly proportional to $P^{(3)}(\omega)$ [28].

As the NIR pulse duration is much shorter than the period of the terahertz field and the frequency dependence of $\chi^{(3)}\left(\omega_{\mathrm{NIR}}, \omega_{\mathrm{NIR}}, \omega_{\mathrm{THz}}\right)$ within the terahertz spectral bandwidth can be neglected in first approximation, we are left with $I_{\mathrm{SHG}} \propto E(t)=V(t) / d$. Here, $E(t)$ is the electric field that develops at the very surface of the material, within an interaction region of $\sim 100 \mathrm{~nm}$ (set by the NIR probe penetration depth). In a Josephson junction, this quantity corresponds to $V(t) / d$, where $V(t)$ is the time-dependent voltage across the junction and $d$ the layer separation. Hence, our technique provides a 

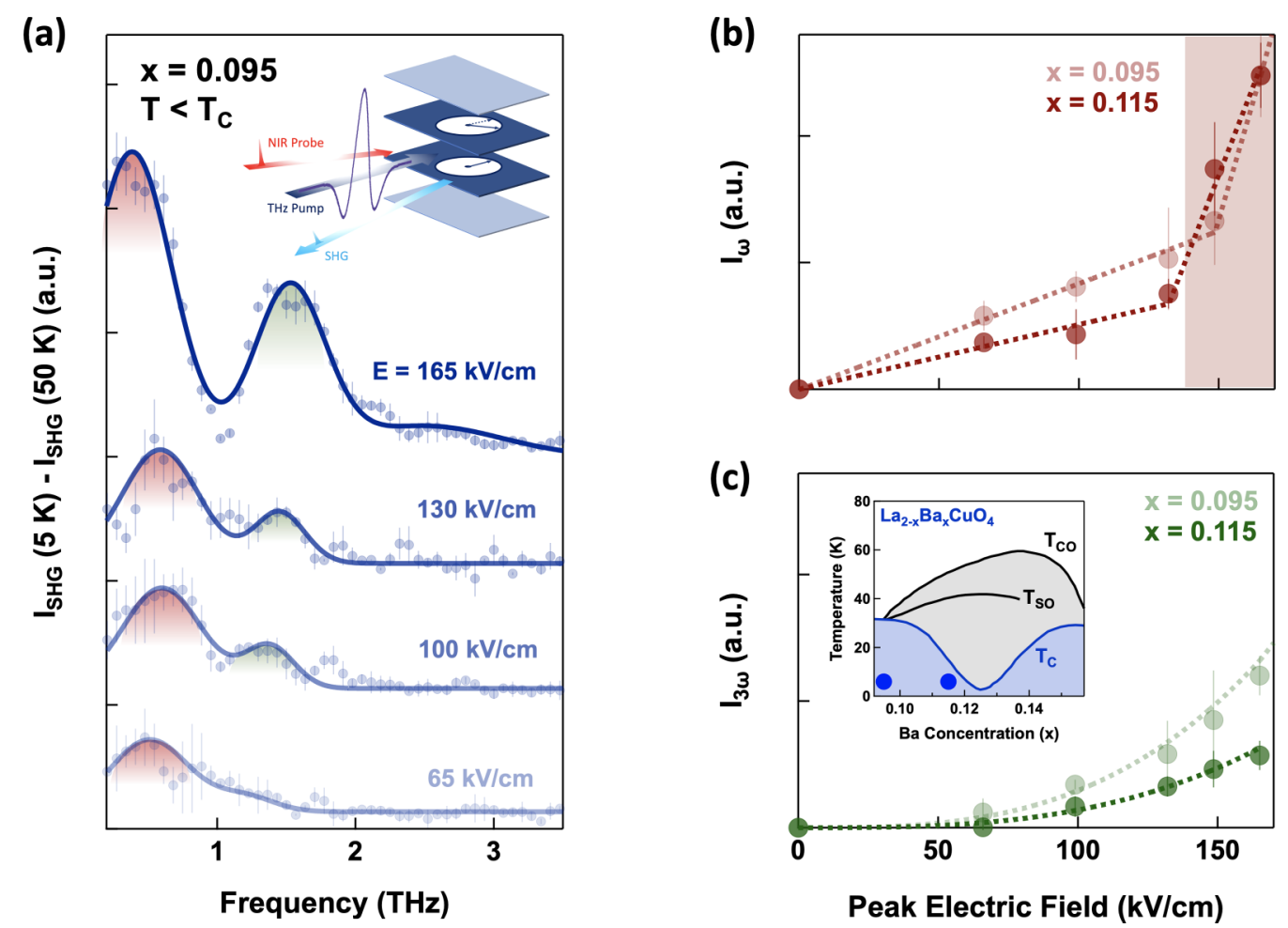

FIG. 2. (a) Fourier transform of the second harmonic intensity measured in superconducting $\mathrm{La}_{1.905} \mathrm{Ba}_{0.095} \mathrm{CuO}_{4}$ at $T=5 \mathrm{~K}<T_{C}$ for different terahertz peak driving fields (see legend), after subtraction of the same quantity measured at $T>T_{C}$. The spectra have been vertically offset, maintaining their relative amplitude. Uncertainty bars are standard errors estimated from different measurement sets. The peaks at $\sim \omega_{\text {drive }}$ and $\sim 3 \omega_{\text {drive }}$ are shaded in red and green, respectively. Inset: Schematic of the experimental geometry, in which a single-cycle terahertz pulse polarized along the $c$ axis is shone onto the sample surface together with a near-infrared (NIR) probe pulse (also polarized along $c$ ). The radiation generated at the second harmonic of the probe is then detected. The clocks in the layers represent amplitude (diameter) and phase (hand angle) of the superconducting order parameter. (b) Light red: Peak electric field dependence of the $\sim \omega_{\text {drive }}$ spectral component. Error bars are extracted here from the multi-Gaussian fits in (a). Dark red: Same quantity, measured in $\mathrm{La}_{1.885} \mathrm{Ba}_{0.115} \mathrm{CuO}_{4}$ at $T=5 \mathrm{~K}<T_{C}$, for which full sets of spectra are reported in Fig. S3(a) in the Supplemental Material [15]. Dashed lines are linear guides to the eye, while the red shading highlights the phase-slip regime. (c) Same quantities as in (b), extracted for the $\sim 3 \omega_{\text {drive }}$ component. Here, the dashed lines are $\propto E^{3}$ fits. The vertical scales in (b) and (c) are mutually calibrated. Inset: Temperature-doping phase diagram of $\mathrm{La}_{2-x} \mathrm{Ba}_{x} \mathrm{CuO}_{4}$, where the exact location of the investigated compounds is indicated by blue circles. Here, $T_{\mathrm{CO}}, T_{\mathrm{SO}}$, and $T_{C}$ are the charge-order, spin-order, and superconducting transition temperature, respectively.

direct experimental measurement of the Josephson voltage at the sample surface, enabling a one-to-one comparison with the calculated quantities in Figs. 1(e) and 1(f).

This type of measurement is different from that reported in Ref. [28]. Therein, coherent oscillations in the second harmonic signal, persisting over much longer time windows than the duration of the pump pulse, were attributed to an effective second-order susceptibility, which tracked the time-dependent oscillations of symmetry-odd, infrared-active modes. In our case, instead, we are dealing with an effect that is present only while the terahertz driving field excites the material and therefore can be fully described in terms of an EFISH process.

In Fig. 2(a), we report the results of the experiment carried out in the superconducting phase at the lowest temperature $(T=5 \mathrm{~K})$ in LBCO 9.5\%. Therein, we show the Fourier transform of the measured EFISH time trace for various peak amplitudes of the terahertz driving field, having subtracted the same quantity measured at high temperature. The exact type of measurements was also performed in the superconducting state of LBCO $11.5 \%$ (see Fig. S3(a) in the Supplemental Material [15]).

In both compounds, the response was almost identical, characterized by a strong peak at the fundamental driving frequency $\omega_{\text {drive }} \simeq 0.5 \mathrm{THz}$ (which in LBCO 9.5\% overlaps with the equilibrium $\omega_{\mathrm{JPR}}$ [29]), whose intensity scaled with the terahertz field amplitude. Concurrently, another contribution developed for $E_{\text {peak }} \gtrsim 100 \mathrm{kV} / \mathrm{cm}$, with oscillations appearing at the third harmonic frequency $3 \omega_{\text {drive }} \simeq 1.5 \mathrm{THz}$, and only for the highest field data $\left(E_{\text {peak }}=165 \mathrm{kV} / \mathrm{cm}\right)$, signatures of a $5 \omega_{\text {drive }} \simeq 2.5 \mathrm{THz}$ peak were also found. The latter, however, is comparable with the noise floor of our measurement, and we refrain from analyzing it further, focusing on the $\omega_{\text {drive }}$ and $3 \omega_{\text {drive }}$ terms.

The frequencies of these contributions did not depend strongly on $E_{\text {peak }}$. We found instead a systematically higher signal amplitude in LBCO $9.5 \%$ compared with LBCO $11.5 \%$, likely related to the detuning of $\omega_{\text {drive }}$ compared with the resonance at $\omega_{\mathrm{JPR}}$, which in the latter material is $\omega_{\mathrm{JPR}} \simeq$ $0.2 \mathrm{THz}$. Note that this frequency was contained in the drive 

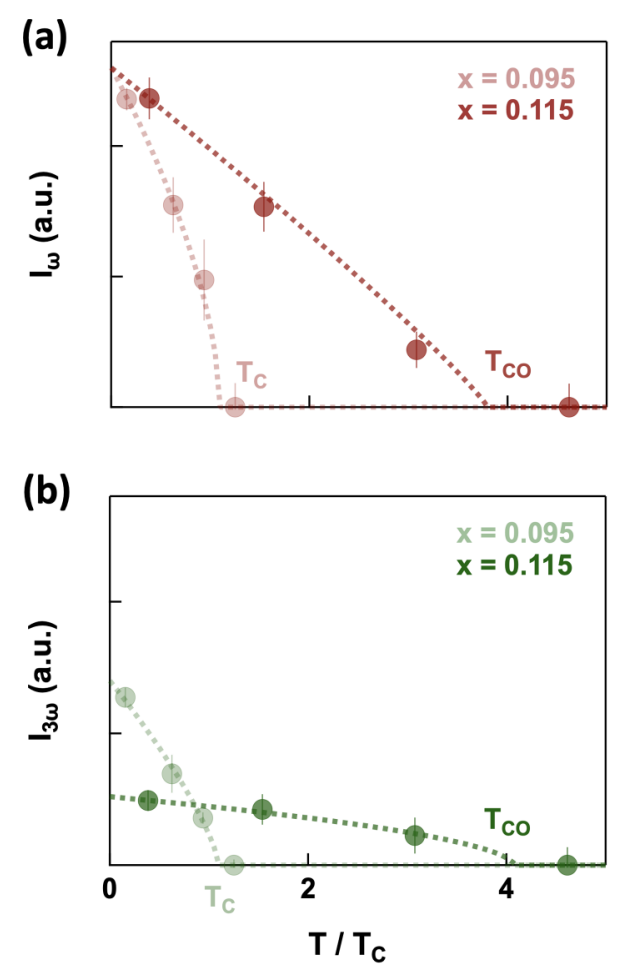
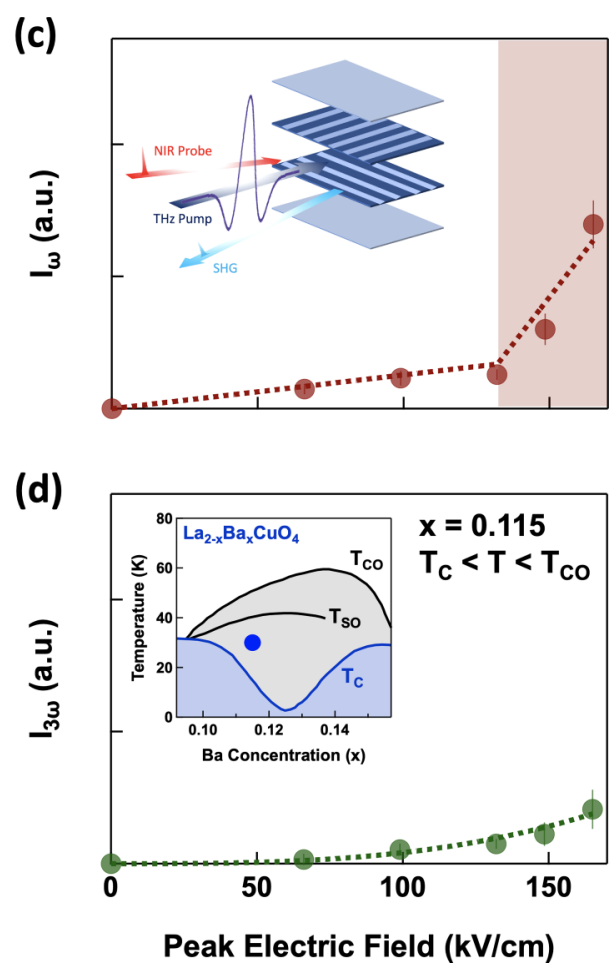

FIG. 3. (a) Temperature dependence of the $\sim \omega_{\text {drive }}$ spectral component in the second harmonic intensity measured for both $\mathrm{La}_{1.905} \mathrm{Ba}_{0.095} \mathrm{CuO}_{4}$ (light red) and $\mathrm{La}_{1.885} \mathrm{Ba}_{0.115} \mathrm{CuO}_{4}$ (dark red), for a constant terahertz peak driving field of $\sim 165 \mathrm{kV} / \mathrm{cm}$. Error bars are extracted from multi-Gaussian fits to the experimental spectra reported in Fig. S4 in the Supplemental Material [15], while dashed lines are mean field fits. The horizontal temperature axis has been normalized by the superconducting $T_{\mathrm{C}}$ of each compound. While for $\mathrm{La}_{1.905} \mathrm{Ba}_{0.095} \mathrm{CuO}_{4}$ the response vanishes at $T_{C}=T_{\mathrm{CO}}=32 \mathrm{~K}$, in $\mathrm{La}_{1.885} \mathrm{Ba}_{0.115} \mathrm{CuO}_{4}$, it persists well above $T_{C}=13 \mathrm{~K}$, all the way up to $\sim T_{\mathrm{CO}}=53 \mathrm{~K}$. (b) Same quantities as in (a), extracted for the $\sim 3 \omega_{\text {drive }}$ component. (c) Peak electric field dependence of the $\sim \omega_{\text {drive }}$ component measured in $\mathrm{La}_{1.885} \mathrm{Ba}_{0.115} \mathrm{CuO}_{4}$ at $T_{C}<T=30 \mathrm{~K}<T_{\mathrm{SO}}, T_{\mathrm{CO}}$, for which full sets of spectra are reported in Fig. S3(b) in the Supplemental Material [15]. Dashed lines are linear guides to the eye, while the red shading highlights the phase-slip regime. Inset: Schematic of the experimental geometry. The charge order pattern in the layers is displayed as colored stripes. (d) Same quantity as in (c), extracted for the $\sim 3 \omega_{\text {drive }}$ component. Here, the dashed lines are $\propto E^{3}$ fits. The vertical scales in (a)-(d) are mutually calibrated. Inset: Temperature-doping phase diagram of $\mathrm{La}_{2-x} \mathrm{Ba}_{x} \mathrm{CuO}_{4}$, where the exact location of the investigated compound is indicated by a blue circle.

spectrum but not at its peak (see Fig. S1 in the Supplemental Material [15]).

Figure 2 reports the $E_{\text {peak }}$ dependence in the EFISH signal of the (b) $\omega_{\text {drive }}$ and (c) $3 \omega_{\text {drive }}$ contributions, estimated from multi-Gaussian fits to the data in Figs. 2(a) and S3(a) in the Supplemental Material [15]. While the third harmonic displays a $\propto E_{\text {peak }}^{3}$ dependence, the fundamental peak shows a linear behavior only for low fields. A clear discontinuity is observed at $\sim 150 \mathrm{kV} / \mathrm{cm}$ in LBCO $9.5 \%$ and $\sim 130 \mathrm{kV} / \mathrm{cm}$ in LBCO $11.5 \%$. This trend is reminiscent of that found in the voltage simulations of Fig. 1(f) and is indicative of Josephson phase slips in the superconducting phase of both materials when sufficiently high transient terahertz fields are applied. It is also not surprising that this effect is observed for slightly higher $E_{\text {peak }}$ values in LBCO 9.5\%, a superconductor with higher critical temperature and larger phase rigidity.

The temperature dependence, which we report for both samples in Figs. 3(a) and 3(b) at a constant $E_{\text {peak }} \simeq 165$ $\mathrm{kV} / \mathrm{cm}$ (see Fig. S4 in the Supplemental Material [15] for the original spectra), provides additional information on these phenomena. While in the weakly striped superconductor LBCO 9.5\% the doubly peaked EFISH response progressively reduces with increasing temperature approaching $T_{C} \simeq T_{\mathrm{SO}} \simeq$ $T_{\mathrm{CO}}$ and then completely disappears in the stripe-free normal state, a much more striking effect is observed in LBCO $11.5 \%$. Here, both the $\omega_{\text {drive }}$ and $3 \omega_{\text {drive }}$ peaks clearly survive at $T>T_{C}$ all the way up to the charge-ordering temperature $T_{\mathrm{CO}}$.

The presence of phase slips in the stripe-ordered normal state of LBCO $11.5 \%$ is further underscored by the data in Figs. 3(c) and 3(d). These figures have the same structure as Figs. 2(b) and 2(c) (see Fig. S3(b) in the Supplemental Material [15] for full spectra), with the difference that the data here were taken at $T=30 \mathrm{~K}$, a temperature almost three times higher than $T_{C}$ in this compound. The response is virtually identical to that measured in the superconducting state, with a clear discontinuity in the field dependence of the fundamental peak [Fig. 3(c)] $\sim 130 \mathrm{kV} / \mathrm{cm}$, a behavior which is very reminiscent of that predicted by the simulation of Fig. 1(f).

The observation of phase slips in the stripe-ordered state of $\mathrm{La}_{1.885} \mathrm{Ba}_{0.115} \mathrm{CuO}_{4}$ complements the colossal third-harmonic signal previously reported in Ref. [19], providing experimental evidence for interlayer coherence and possibly for finite 
momentum condensation in the normal state of this cuprate. While we can associate the voltage anomalies with phase slips in a finite momentum condensate, a comprehensive theory for this phenomenon has not yet been formulated.

A natural evolution for this field of study should address other forms of charge order that compete or coexist with superconductivity, such as those found in $\mathrm{YBa}_{2} \mathrm{Cu}_{3} \mathrm{O}_{6+x}$ [30,31]. This same technique also has great potential to be applied in other regions of the cuprate phase diagram, where for example finite superfluid density and vanishing range phase correlations [32,33] are present, or other forms of density waves [34,35] have been discussed.
The research leading to these results received funding from the European Research Council (ERC) under the European Union's Seventh Framework Programme (No. FP7/20072013)/ERC Grant Agreement No. 319286 (QMAC). We acknowledge support from the Deutsche Forschungsgemeinschaft via the excellence cluster The Hamburg Centre for U1trafast Imaging (EXC 1074-Project ID No. 194651731) and the priority program SFB925 (Project ID No. 170620586). Work at Brookhaven is supported by the Office of Basic Energy Sciences, Division of Materials Sciences and Engineering, U.S. Department of Energy under Contract No. DE-SC0012704.
[1] W. E. Lawrence and S. Doniach, in Proceedings of the Twelfth International Conference on Low Temperature Physics, edited by E. Kanda (Tokyo Keigaku Publishing, Kyoto, 1971), pp. 361-362.

[2] R. Kleiner, F. Steinmeyer, G. Kunkel, and P. Müller, Intrinsic Josephson Effects in $\mathrm{Bi}_{2} \mathrm{Sr}_{2} \mathrm{CaCu}_{2} \mathrm{O}_{8}$ Single Crystals, Phys. Rev. Lett. 68, 2394 (1992).

[3] O. K. C. Tsui, N. P. Ong, Y. Matsuda, Y. F. Yan, and J. B. Peterson, Sharp Magnetoabsorption Resonances in the Vortex State of $\mathrm{Bi}_{2} \mathrm{Sr}_{2} \mathrm{CaCu}_{2} \mathrm{O}_{8+\delta}$, Phys. Rev. Lett. 73, 724 (1994).

[4] Y. Matsuda, M. B. Gaifullin, K. Kumagai, K. Kadowaki, and T. Mochiku, Collective Josephson Plasma Resonance in the Vortex State of $\mathrm{Bi}_{2} \mathrm{Sr}_{2} \mathrm{CaCu}_{2} \mathrm{O}_{8+\delta}$, Phys. Rev. Lett. 75, 4512 (1995).

[5] O. K. C. Tsui, N. P. Ong, and J. B. Peterson, Excitation of the Josephson Plasma Mode in $\mathrm{Bi}_{2} \mathrm{Sr}_{2} \mathrm{CaCu}_{2} \mathrm{O}_{8+\delta}$ in an Oblique Field, Phys. Rev. Lett. 76, 819 (1996).

[6] K. Tamasaku, Y. Nakamura, and S. Uchida, Charge Dynamics across the $\mathrm{CuO}_{2}$ Planes in $\mathrm{La}_{2-x} \mathrm{Sr}_{x} \mathrm{CuO}_{4}$, Phys. Rev. Lett. 69, 1455 (1992).

[7] S. Savel'ev, A. L. Rakhmanov, V. A. Yampol'skii, and F. Nori, Analogues of nonlinear optics using terahertz Josephson plasma waves in layered superconductors, Nature Phys. 2, 521 (2006).

[8] S. Savel'ev, V. A. Yampol'skii, A. L. Rakhmanov, and F. Nori, Terahertz Josephson plasma waves in layered superconductors: spectrum, generation, nonlinear and quantum phenomena, Rep. Prog. Phys. 73, 026501 (2010).

[9] Y. Laplace and A. Cavalleri, Josephson plasmonics in layered superconductors, Adv. Phys.: X 1, 387 (2016).

[10] M. Tinkham, Introduction to Superconductivity (McGraw-Hill, New York, 1996).

[11] D. Stornaiuolo, G. Rotoli, D. Massarotti, F. Carillo, L. Longobardi, F. Beltram, and F. Tafuri, Resolving the effects of frequency dependent damping and quantum phase diffusion in $\mathrm{YBa}_{2} \mathrm{Cu}_{3} \mathrm{O}_{7-x}$ Josephson junctions, Phys. Rev. B 87, 134517 (2013).

[12] A. Barone and G. Paternò, Physics and Applications of Josephson Effect (Wiley, New York, 1982).

[13] M. H. Devoret, J. M. Martinis, and J. Clarke, Measurements of Macroscopic Quantum Tunneling out of the Zero-Voltage State of a Current-Biased Josephson Junction, Phys. Rev. Lett. 55, 1908 (1985).

[14] X. Hu and S. Z. Lin, Phase dynamics in a stack of inductively coupled intrinsic Josephson junctions and terahertz electromagnetic radiation, Supercond. Sci. Technol. 23, 053001 (2010).
[15] See Supplemental Material at http://link.aps.org/supplemental/ 10.1103/PhysRevB.105.L020502 for simulation of the Josephson phase dynamics with the sine-Gordon equation, experimental methods, and additional datasets, which includes Refs. $[18,19,26,27,29,36,37]$.

[16] A. Dienst, M. C. Hoffmann, D. Fausti, J. C. Petersen, S. Pyon, T. Takayama, H. Takagi, and A. Cavalleri, Bi-directional ultrafast electric-field gating of interlayer charge transport in a cuprate superconductor, Nat. Photon. 5, 485 (2011).

[17] A. Dienst, E. Casandruc, D. Fausti, L. Zhang, M. Eckstein, M. Hoffmann, V. Khanna, N. Dean, M. Gensch, S. Winnerl, W. Seidel, S. Pyon, T. Takayama, H. Takagi, and A. Cavalleri, Optical excitation of Josephson plasma solitons in a cuprate superconductor, Nat. Mater. 12, 535 (2013).

[18] S. Rajasekaran, E. Casandruc, Y. Laplace, D. Nicoletti, G. D. Gu, S. R. Clark, D. Jaksch, and A. Cavalleri, Parametric amplification of a superconducting plasma wave, Nat. Phys. 12, 1012 (2016).

[19] S. Rajasekaran, J. Okamoto, L. Mathey, M. Fechner, V. Thampy, G. D. Gu, and A. Cavalleri, Probing optically silent superfluid stripes in cuprates, Science 359, 575 (2018).

[20] M. Hücker, M. v. Zimmermann, G. D. Gu, Z. J. Xu, J. S. Wen, G. Xu, H. J. Kang, A. Zheludev, and J. M. Tranquada, Stripe order in superconducting $\mathrm{La}_{2-x} \mathrm{Ba}_{x} \mathrm{CuO}_{4}(0.095 \leqslant x \leqslant 0.155)$, Phys. Rev. B 83, 104506 (2011).

[21] J. M. Tranquada, B. J. Sternlieb, J. D. Axe, Y. Nakamura, and S. Uchida, Evidence for stripe correlations of spins and holes in copper oxide superconductors, Nature (London) 375, 561 (1995).

[22] Q. Li, M. Hücker, G. D. Gu, A. M. Tsvelik, and J. M. Tranquada, Two-Dimensional Superconducting Fluctuations in Stripe-Ordered $\mathrm{La}_{1.875} \mathrm{Ba}_{0.125} \mathrm{CuO}_{4}$, Phys. Rev. Lett. 99, 067001 (2007).

[23] E. Berg, E. Fradkin, E.-A. Kim, S. A. Kivelson, V. Oganesyan, J. M. Tranquada, and S. C. Zhang, Dynamical Layer Decoupling in a Stripe-Ordered High- $T_{\mathrm{C}}$ Superconductor, Phys. Rev. Lett. 99, 127003 (2007).

[24] E. Berg, E. Fradkin, S. A. Kivelson, and J. M. Tranquada, Striped superconductors: how spin, charge and superconducting orders intertwine in the cuprates, New J. Phys. 11, 115004 (2009).

[25] D. F. Agterberg, J. C. Séamus Davis, S. D. Edkins, E. Fradkin, D. J. Van Harlingen, S. A. Kivelson, P. A. Lee, L. Radzihovsky, J. M. Tranquada, and Y. Wang, The physics of 
pair-density waves: cuprate superconductors and beyond, Annu. Rev. Condens. Matter Phys. 11, 231 (2020).

[26] J. Hebling, K.-L. Yeh, M. C. Hoffmann, B. Bartal, and K. A. Nelson, Generation of high-power terahertz pulses by tiltedpulse-front excitation and their application possibilities, J. Opt. Soc. Am. B 25, B6 (2008).

[27] M. Fiebig, V. V. Pavlov, and R. V. Pisarev, Second-harmonic generation as a tool for studying electronic and magnetic structures of crystals: review, J. Opt. Soc. Am. B 22, 96 (2005).

[28] A. von Hoegen, M. Fechner, M. Först, N. Taherian, E. Rowe, A. Riback, J. Porras, B. Keimer, M. Michael, E. Demler, and A. Cavalleri, Parametrically amplified phase-incoherent superconductivity in $\mathrm{YBa}_{2} \mathrm{Cu}_{3} \mathrm{O}_{6+x}$, arXiv:1911.08284 (2019).

[29] C. C. Homes, M. Hücker, Q. Li, Z. J. Xu, J. S. Wen, G. D. Gu, and J. M. Tranquada, Determination of the optical properties of $\mathrm{La}_{2-x} \mathrm{Ba}_{x} \mathrm{CuO}_{4}$ for several dopings, including the anomalous $x=\frac{1}{8}$ phase, Phys. Rev. B 85, 134510 (2012).

[30] G. Ghiringhelli, M. Le Tacon, M. Minola, S. Blanco-Canosa, C. Mazzoli, N. B. Brookes, G. M. De Luca, A. Frano, D. G. Hawthorn, F. He, T. Loew, M. Moretti Sala, D. C. Peets, M. Salluzzo, E. Schierle, R. Sutarto, G. A. Sawatzky, E. Weschke, B. Keimer, and L. Braicovich, Long-range incommensurate charge fluctuations in $(\mathrm{Y}, \mathrm{Nd}) \mathrm{Ba}_{2} \mathrm{Cu}_{3} \mathrm{O}_{6+x}$, Science 337, 821 (2012).

[31] J. Chang, E. Blackburn, A. T. Holmes, N. B. Christensen, J. Larsen, J. Mesot, Ruixing Liang, D. A. Bonn, W. N. Hardy, A. Watenphul, M. v. Zimmermann, E. M. Forgan, and S. M.
Hayden, Direct observation of competition between superconductivity and charge density wave order in $\mathrm{YBa}_{2} \mathrm{Cu}_{3} \mathrm{O}_{6.67}$, Nat. Phys. 8, 871 (2012).

[32] J. Corson, R. Mallozzi, J. Orenstein, J. N. Eckstein, and I. Bozovic, Vanishing of phase coherence in underdoped $\mathrm{Bi}_{2} \mathrm{Sr}_{2} \mathrm{CaCu}_{2} \mathrm{O}_{8+\delta}$, Nature (London) 398, 221 (1999).

[33] L. S. Bilbro, R. Valdés Aguilar, G. Logvenov, O. Pelleg, I. Bozović, and N. P. Armitage, Temporal correlations of superconductivity above the transition temperature in $\mathrm{La}_{2-x} \mathrm{Sr}_{x} \mathrm{CuO}_{4}$ probed by terahertz spectroscopy, Nat. Phys. 7, 298 (2011).

[34] K. Fujita, M. H. Hamidian, S. D. Edkins, C. K. Kim, Y. Kohsaka, M. Azuma, M. Takano, H. Takagi, H. Eisaki, S. Uchida, A. Allais, M. J. Lawler, E.-A. Kim, S. Sachdev, and J. C. Séamus Davis, Direct phase-sensitive identification of a $d$-form factor density wave in underdoped cuprates, Proc. Natl. Acad. Sci. USA 111, E3026 (2014).

[35] P. A. Lee, Amperean Pairing and the Pseudogap Phase of Cuprate Superconductors, Phys. Rev. X 4, 031017 (2014).

[36] D. Nicoletti, E. Casandruc, Y. Laplace, V. Khanna, C. R. Hunt, S. Kaiser, S. S. Dhesi, G. D. Gu, J. P. Hill, and A. Cavalleri, Optically-induced superconductivity in striped $\mathrm{La}_{2-x} \mathrm{Ba}_{x} \mathrm{CuO}_{4}$ by polarization-selective excitation in the near infrared, Phys. Rev. B 90, 100503(R) (2014).

[37] D. Nicoletti, D. Fu, O. Mehio, S. Moore, A. S. Disa, G. D. $\mathrm{Gu}$, and A. Cavalleri, Magnetic-Field Tuning of Light-Induced Superconductivity in Striped $\mathrm{La}_{2-x} \mathrm{Ba}_{x} \mathrm{CuO}_{4}$, Phys. Rev. Lett. 121, 267003 (2018). 